\title{
To Cast, to Saw, and Not to Injure: Can Safety Strips Decrease Cast Saw Injuries?
}

\author{
Natalie C. Stork MD, Rachel L. Lenhart PhD, Blaise A. Nemeth MD, MS, \\ Kenneth J. Noonan MD, Matthew A. Halanski MD
}

Received: 24 July 2015/ Accepted: 21 January 2016/Published online: 4 February 2016

(C) The Association of Bone and Joint Surgeons (B) 2016

\begin{abstract}
Background Placement and removal of fiberglass casts are among the more-common interventions performed in pediatric orthopaedic surgery offices. However, cast removal is associated with abrasive injuries and burns from the oscillating cast saw, and these injuries can occur even when the cast is removed by experienced personnel. It is unknown whether an added barrier, such as a safety strip, can mitigate injuries from blade-to-skin contact during cast removal with the oscillating saw.

Questions/purposes We asked: (1) Can a safety strip provide a physical barrier during cast removal, decreasing
\end{abstract}

Each author certifies that he or she or a member of his or her immediate family, has no funding or commercial associations (eg, consultancies, stock ownership, equity interest, patent/licensing arrangements, etc) that might pose a conflict of interest in connection with the submitted article.

All ICMJE Conflict of Interest Forms for authors and Clinical Orthopaedics and Related Research ${ }^{\circledR}$ editors and board members are on file with the publication and can be viewed on request.

Clinical Orthopaedics and Related Research ${ }^{\mathbb{R}}$ neither advocates nor endorses the use of any treatment, drug, or device. Readers are encouraged to always seek additional information, including FDAapproval status, of any drug or device prior to clinical use.

This study was performed at the University of Wisconsin, Madison, WI, USA.

\section{N. C. Stork}

Departments of Orthopaedic Surgery and Pediatrics, University of Missouri-Kansas City School of Medicine, Kansas City, MO, USA

\section{N. C. Stork $(\square)$}

The Division of Orthopaedics, Section of Sports Medicine, Children's Mercy Kansas City, 2401 Gillham Road, Kansas City, MO 64108, USA

e-mail: ncstork@cmh.edu blade-to-skin contact? (2) Does the safety strip lessen heat transfer? (3) Will the use of the safety strip prevent cast pressure from being released when the cast is split?

Methods Standard long-arm fiberglass casts were removed by experienced and inexperienced healthcare personnel $(\mathrm{n}=35)$ from life-sized pediatric models. A commercially available woven cast saw safety strip, commonly incorporated in waterproof cast constructs, was chosen as the protective strip. Each participant removed a cast with and without the safety strip present. All participants were blinded to the presence or absence of the safety strip at the time of cast removal. The number of touches was compared between cast removal with and without protective strips. A separate model was designed to assess prevention of heat transfer. Temperatures were recorded, using thermocouples, for three designated temperatures. Five to six trials were conducted at each designated temperature for each of two conditions, with and without the safety strip. Finally, to assess if the safety strip would prevent cast pressure from being released, a third model was used. Thirty standard short-arm casts were applied and removed from the arm models by one of the authors. Pressure data were collected from between the padding layers, in casts with and without the safety strip present, after application, univalving and bivalving each cast.

\section{R. L. Lenhart}

University of Wisconsin School of Medicine and Public Health, Madison, WI, USA

B. A. Nemeth, K. J. Noonan, M. A. Halanski Department of Orthopedics and Rehabilitation, University of Wisconsin School of Medicine and Public Health, Madison, WI, USA 
Results Use of the safety strip reduced the number of simulated skin touches compared with casts removed without the safety strip, among experienced users (mean, 9.0 [range, 1-28] versus 0.1 [range, 0-1], mean ratio, 0.0012; 95\% CI, 0.002-0.063; p < 0.001) and inexperienced users (mean, 8.5 [range, $0-31$ ] versus 0.6 [range, $0-$ 3], mean ratio, 0.07; 95\% CI, 0.03-0.15; p $<0.001)$. The safety strips decreased heat transfer, preventing temperatures at the cast-skin interface from reaching $50{ }^{\circ} \mathrm{C}$. Finally, after splitting the cast, with the numbers available, there was no increase in the pressure beneath the casts in those with the safety strip present (mean without, 0.23 [SD, 0.070] versus safety strip in the padding 0.20 [SD, 0.091] and safety strip on top padding, 0.21 [SD, 0.090]; $p=0.446$ and $\mathrm{p}=0.65$ respectively).

Conclusions Our study showed the effectiveness of a safety strip in reducing simulated touches with the oscillating cast saw during cast splitting. Additional studies are warranted to investigate the clinical use and utility of the safety strip in practice.

Clinical Relevance The findings of this study suggest that using safety strips in clinical practice could decrease blade-to-skin contact and therefore minimize cast saw injuries. However, validation of these findings in the clinical setting is necessary before drawing a definitive conclusion.

\section{Introduction}

Oscillating cast saws are commonly used in orthopaedic clinics. Despite a design engineered to cut through hard cast material, sparing the soft padding and epidermis, the incidence of cast saw injuries is between $0.1 \%$ and $0.72 \%$ $[1,12]$. In addition to direct patient harm, these injuries can have considerable indirect costs; costs to one hospital from injuries sustained during cast removal averaged USD 445,144 or USD 15,898 per patient for one year [1, 13]. Cast saw injuries result from thermal and/or abrasive damage to the skin [1, 5, 6, 12, 13]. Shuler and Grisafi [13] reported an average cast saw blade temperature of $56{ }^{\circ} \mathrm{C}$ during removal of fiberglass casts. However, temperatures from $41^{\circ}$ to $102{ }^{\circ} \mathrm{C}$ have been reported [6]. Because permanent dermal injury can occur at temperatures greater than $50{ }^{\circ} \mathrm{C}$, thermal injury during cast removal can pose a real risk [4, 10]. Despite some studies focused on mitigating cast saw blade temperatures $[6,11,13]$, Ansari et al. [1] found the frequency of abrasions was greater than the incidence of burns. This suggests that blade-to-skin contact plays an important role in cast saw injuries that is not well understood. Therefore, prevention of blade-to-skin contact may be the most effective method of decreasing iatrogenic cast saw injuries.

Although some approaches to mitigate the frequency and severity of injuries from the oscillating saw during cast removal have been studied [6, 12], one study showed blade-to-skin contact commonly occurs during cast removal independent of user experience level [9]. We believe that education and training alone, although helpful, may not be enough. Any physical barrier to protect the skin should not increase thermal transfer and should not inhibit the release of pressure on bivalving the cast. To our knowledge, the concept of a protective strip placed under the cast in line with where the cast saw might be used, and which might protect the skin from abrasive or thermal injury, has not been evaluated.

The purpose of our study was to evaluate the adequacy of a safety strip to provide a physical barrier during cast removal. The study was designed to answer three questions: (1) Can the safety strip decrease the number of bladeto-skin touches during cast removal? (2) Will the protective strip lessen heat transfer from the blade to the skin surface? (3) Will the use of the safety strip prevent cast pressure from being released when the cast is split?

\section{Materials and Methods}

\section{Experimental Overview}

The study protocol was reviewed by the university's institutional review board and granted exemption. Three unique models were developed to investigate (1) blade-toskin contact events; (2) heat transfer; and (3) release of pressure on splitting the cast.

\section{Blade-to-skin Contact Model Development}

Six pediatric life-sized arm models, based on a plaster mold of a 9-year-old girl, were constructed of silicone rubber gel (PlatSil ${ }^{\circledR}$ Gel-10; Polytek Development Corporation, Easton, PA, USA) that mimics the consistency of living tissue. Quarter-inch polyvinyl chloride pipe was suspended in the model to provide a bony structure similar to that of the human arm. Copper foil was applied, in a strip fashion, to the volar and dorsal aspects of each upper extremity model (Fig. 1).

The models were designed for use with a dedicated cast saw (Zimmer Inc, Warsaw, IN, USA) attached to a DC power source and a voltage data logger (MadgeTech Inc, Warner, NH, USA). When the cast saw blade touched the 


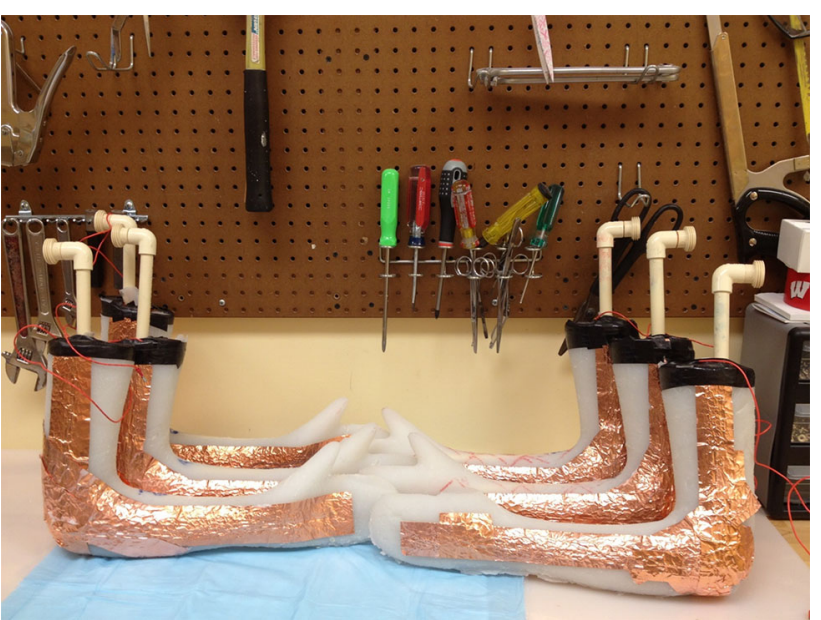

Fig. 1 Six pediatric upper extremity models are shown

model's foil skin, the DC circuit was completed and the change in voltage recorded (Fig. 2).

All casts were applied by one of the authors who was experienced in cast application (NCS), using $3 \mathrm{M}^{\mathrm{TM}}$ fiberglass casting tape (3M Corporation, St Paul, MN, USA) submersed in cool water, just before application. Owing to the conductive nature of water, the voltage at which wet cotton padding conducted electricity was identified to eliminate "false" touches that might be generated through wet padding. To establish this threshold voltage, data were collected separately after application of two and four layers of wet cotton padding with and without a wet safety strip. The wet casting materials conducted to a maximum voltage of $1.88 \mathrm{~V}$. Thus, the threshold voltage for a blade-to-skin touch was greater than $1.90 \mathrm{~V}$.

A power analysis showed that a blinded, paired design with 40 subjects, each removing two casts, one with and one without a safety strip, would have $80 \%$ power in a paired t-test with two-sided alpha 0.05 if the true effect of the safety strip was to decrease the number of touches on average by half. Owing to time constraints of the study period, the full number of subjects was not obtained. All analyses were performed after completion of data collection.

\section{Cast Placement and Removal}

Thirty-five participants were recruited from various medical backgrounds. Twenty-five healthcare personnel were inexperienced. Participants were included in this group if their clinical or daily activities did not involve routine removal of casts with a cast saw. Ten healthcare personnel from the orthopaedic department whose clinical activities involved the routine use of a cast saw, comprised the experienced participants.

Long-arm fiberglass casts were applied in a standard fashion (four layers of padding and six- to eight-ply cast thickness), to each model, by one of the authors (NCS). AquaCast $^{\mathrm{TM}}$ Saw Stop ${ }^{\mathrm{TM}}$ Protective Strips (AquaCast Liner LLC, Middletown, DE, USA) safety strips, an inch in width, were incorporated in each cast construct designated as a safety strip-containing cast. The medial and lateral
Fig. 2A-B (A) The blade-to-skin contact model has an incomplete DC circuit where the cast saw blade is not in contact with the model and no voltage is recorded. (B) When the cast saw blade contacts the arm model, the DC circuit is completed and a voltage is recorded.
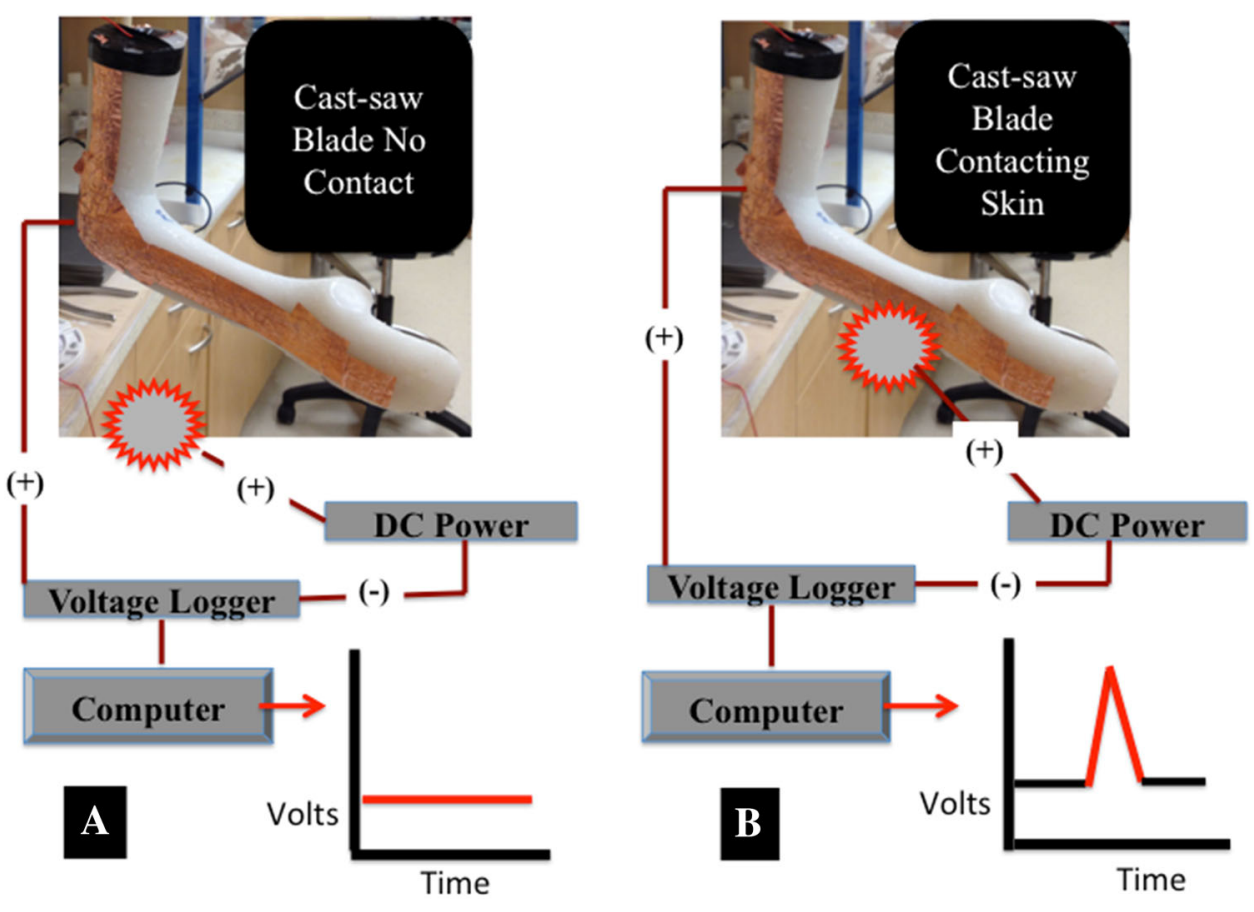


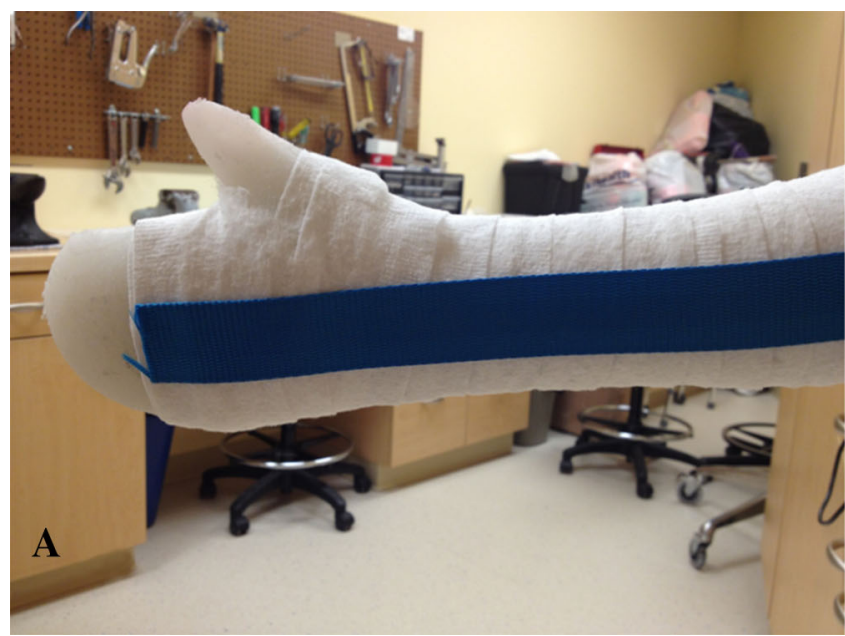

Fig. 3A-B (A) The safety strip was placed on top of four layers of padding before application of casting tape. (B) The safety strip was placed between four layers of padding: two layers are applied over the

aspects of the upper arm each had one longitudinally placed safety strip as did the volar and dorsal aspects of the forearm. Safety strips were placed over the projected path of the cast saw during removal, forming a continuous path. Each safety strip end was incorporated in the cast, so it was not visible, thus blinding the participants to the presence or absence of the strip. Lines drawn on the cast indicated the desired path of the cast saw during removal.

Inexperienced participants were given brief verbal instructions on basic cast saw operation. The order of cast removal for the two (inexperienced) or three conditions (experienced) was randomized by a uniformly distributed number generator so that each possibility was used equally. Each participant was blinded to the presence or absence of the safety strip on cast removal. Participants were asked to cut along the predrawn lines using proper technique by alternating firm pressure against the hard cast material, with relaxation and withdrawal of the cast saw from the cast material, as the base layer of fiberglass was breached. This was followed by placement of the cast saw blade at a different location in continuity with the path of removal. Participants chose the side to initiate cutting. Before transitioning to the second side, the initial side was checked, by one of the authors (NCS), with a cast splitter to ensure an effective cut had been made.

Each participant in the inexperienced group removed two casts, one with and one without the safety strip. Five participants removed casts with the safety strip placed on top of four layers of padding (Fig. 3A), and 20 participants removed casts with the safety strip placed in the middle of the padding layers (Fig. 3B). Each experienced participant removed three casts, one without the safety strip, one with the safety strip on top of four layers of padding, and one with the safety strip between the padding layers. After the

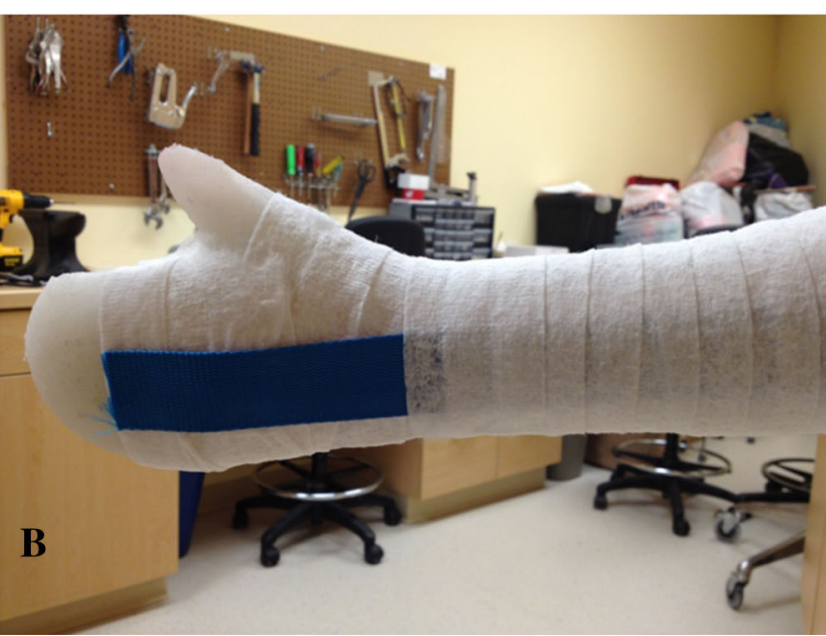

skin, the safety strip is positioned, and then overwrapped with another two layers of padding before application of casting tape.

initial participants successfully cut through the safety strip, concern was raised regarding the role of the location of the safety strip. Because fiberglass becomes sticky when wet, when placed directly on top of the safety strip, there was concern that the strip might adhere, becoming partially incorporated in the casting tape, making the safety strip easier to cut. However, when placed between the layers of cotton, the strip instead serves as a second, independent barrier. Thus, both conditions were assessed.

Cast saw blades were rotated $180^{\circ}$, or changed if previously rotated, after 10 casts had been removed. Voltage measurements were recorded at $4 \mathrm{~Hz}$ and voltages greater than $1.90 \mathrm{~V}$ were identified as touches. The total number of touches for each cast removed and touches lasting a second or greater were analyzed. A mixed-effects negative binomial log-linear regression model was used to analyze the number of touches by strip condition and experience level.

\section{Thermal Conduction Model}

We also wished to test the ability of the safety strip to decrease heat transfer from the cast saw blade to the skin. A cast saw blade was attached to a soldering iron connected to a voltage converter. The temperature of the blade was adjusted to a steady temperature by changing the voltage.

Three t-type thermocouples (Omega Engineering Inc, Stamford, CT, USA) collected temperature data on a model designed to imitate the standard cast construct (Fig. 4). Temperature measurements were recorded for two conditions: three layers of cotton padding with the safety strip and four layers of cotton padding without the safety strip. Three target blade temperature ranges were assessed for 

model with the safety strip present is shown. The heat source is a soldering iron. $\mathrm{T} 1$ is the temperature recorded at the center of the blade, T2 is the temperature recorded at the safety strip-cast saw interface, and T3 is the temperature recorded at the padding-block interface.
Fig. 4 The thermal conduction

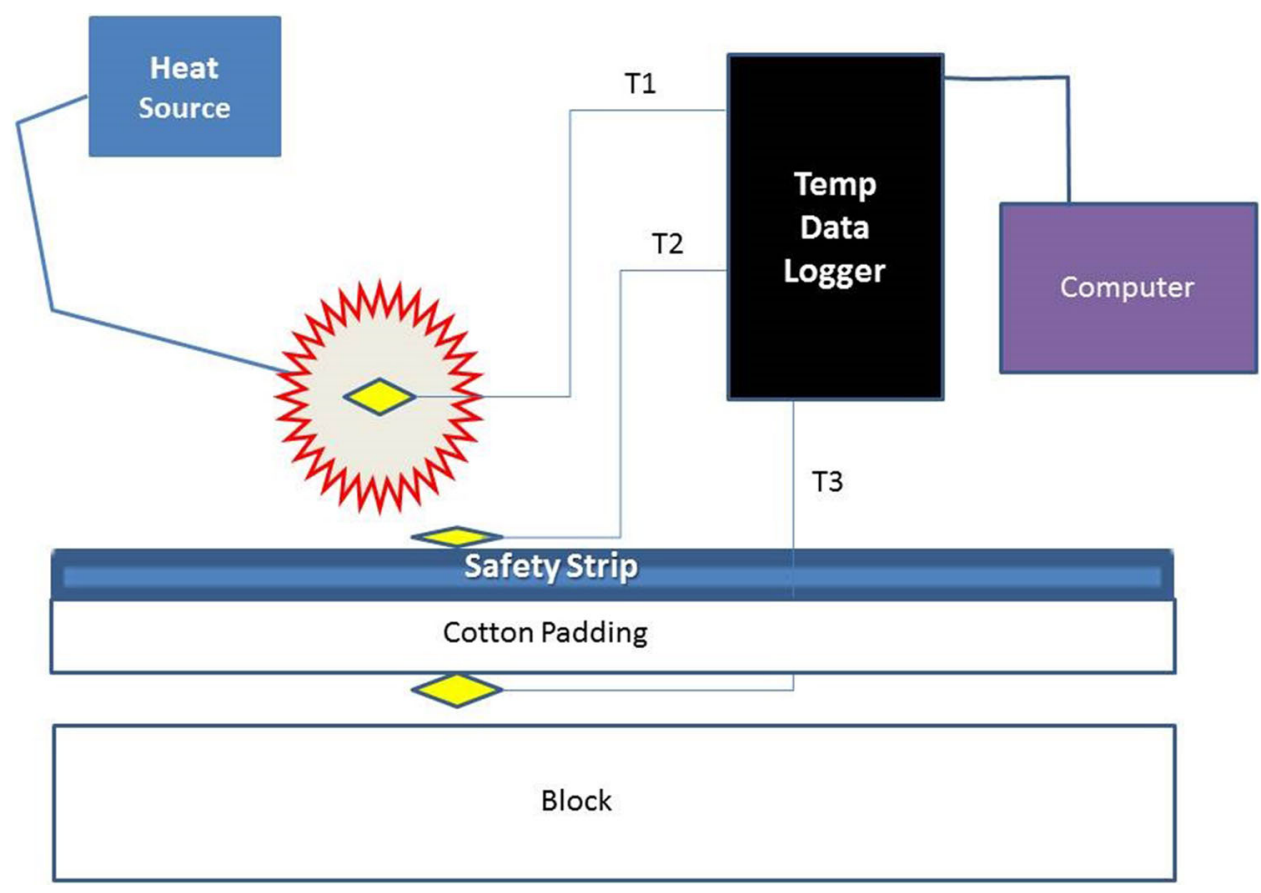

Fig. 5A-D (A) The diagram represents an axial view of the arm model. The two blue rectangles on either side of the arm represent the safety strip, and the rectangle at the superior border of the arm represents one of three forcesensing resistors. (B) The red diamonds on the model represent force-sensing resistor placement on the radial aspect of the model. (C) Top and (D) bottom views of the model show splitting of the cast.

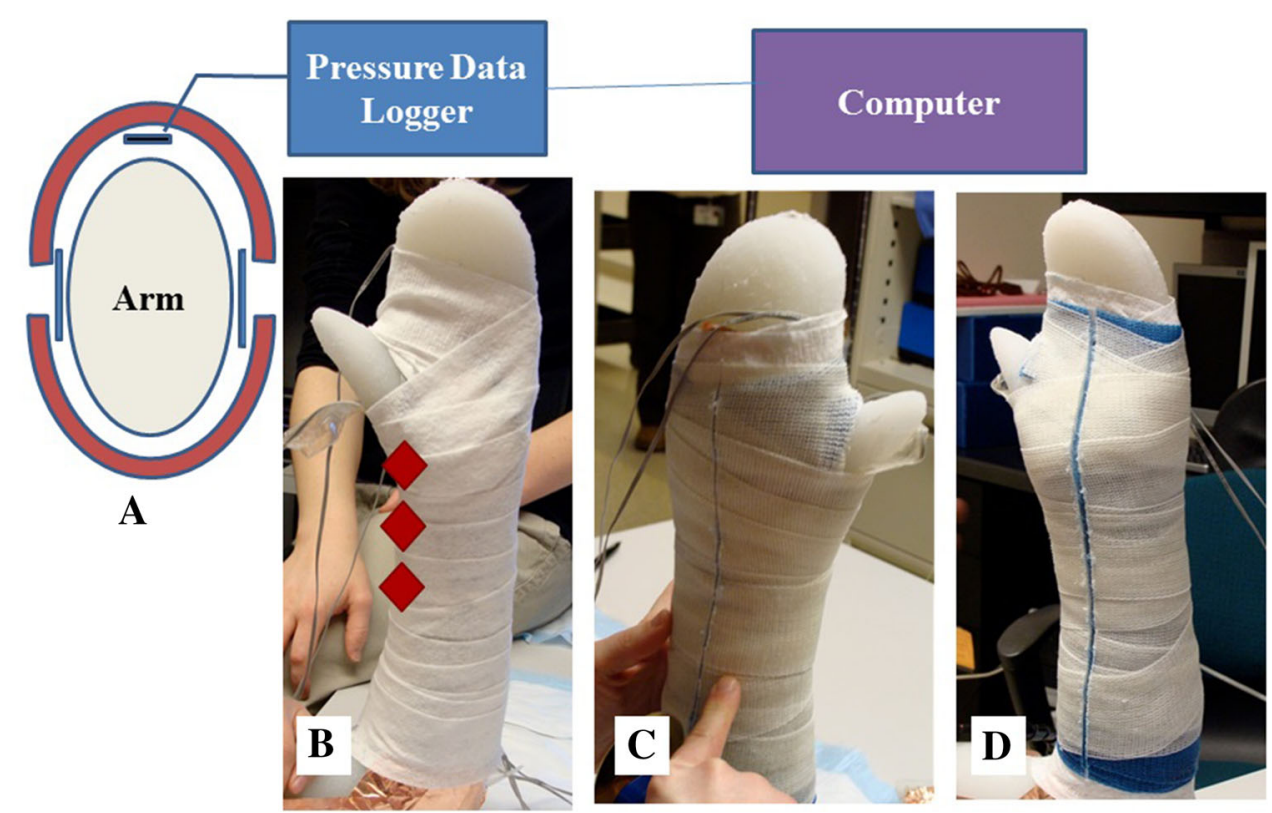

each of the two conditions: $50{ }^{\circ} \mathrm{C}, 65^{\circ}$ to $75^{\circ} \mathrm{C}$, and 100 ${ }^{\circ} \mathrm{C}$. Five to six trials were conducted for each condition at each temperature tested.

The blade remained in contact for an average of 94 seconds per trial. A similar duration between trials allowed the setup to return to baseline temperature. Data were collected continuously at $0.5 \mathrm{~Hz}$. Maximum temperatures at each interface were identified for each trial. The mean (SD) of the five highest, consecutive temperatures surrounding each peak was calculated. Temporal temperature data were aligned and described with best-fit curves for graphic representation and comparison with other data on epidermal injury $[4,10]$.

\section{Pressure Measurement}

We thought it was important that any physical barrier potentially added to the cast construct would not inhibit spreading of the cast when the case was split. Therefore, we wished to determine if the safety strip would prevent release of pressure under the cast after univalving and 
Fig. 6 The mean touches based on condition are shown. The presence of the safety strip decreased the blade-to-skin contact events. *Significant at 0.05 , **significant at 0.001 .

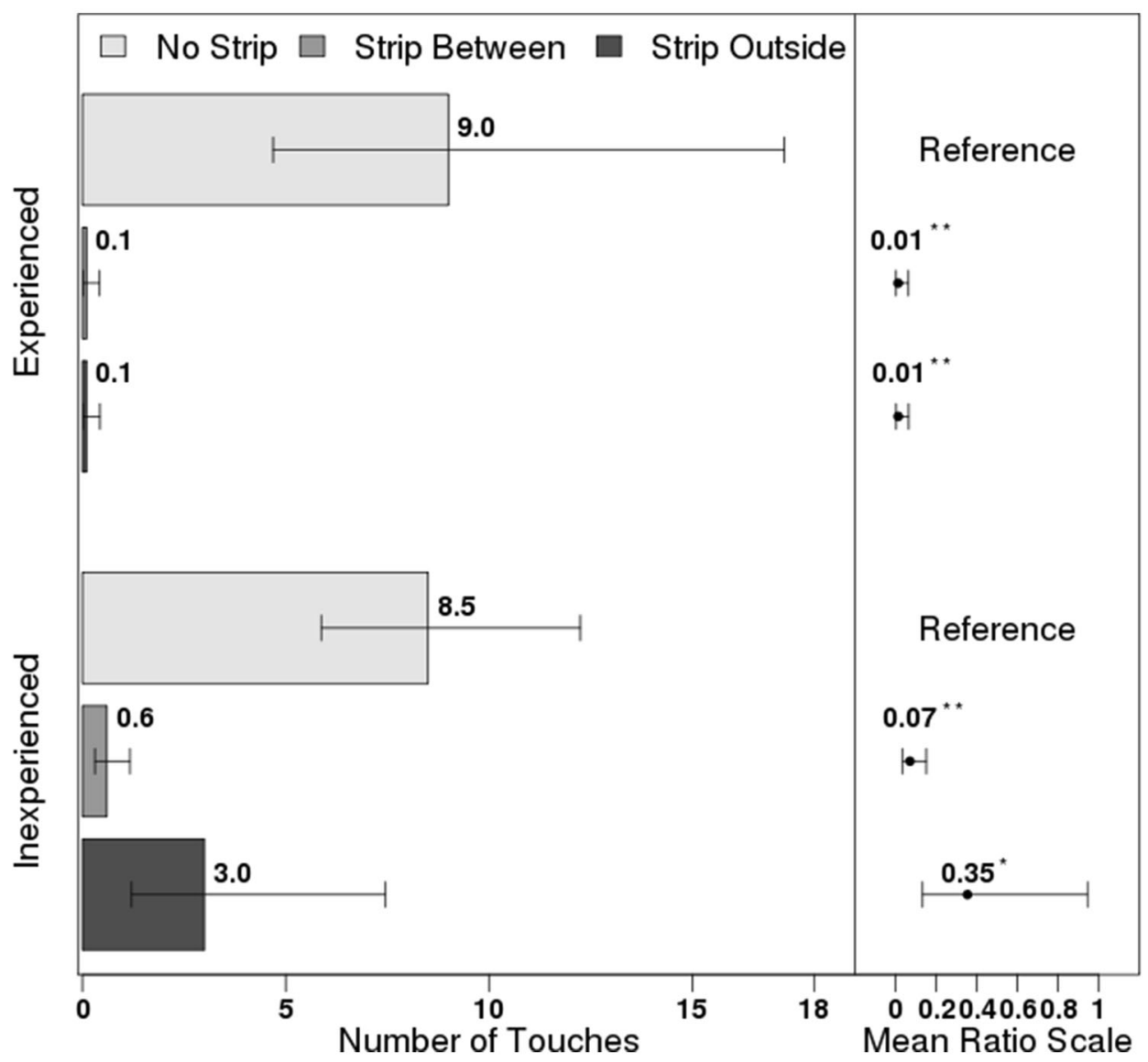

bivalving the cast. Force sensing resistors were incorporated in the cast construct to assess the effect of the safety strip on cast pressure after univalving and bivalving the cast. Data were collected from six 1.5-inch square forcesensing resistors (Interlink Electronics, Camarillo, CA, USA) at $15 \mathrm{~Hz}$. Three adjacent force-sensing resistors were placed on the volar aspect of the forearm and covered with four layers of padding, whereas three force-sensing resistors were placed over the radial aspect in the middle of the padding layers (Fig. 5). Only the force-sensing resistors on the radial aspect were used for analysis as a result of intermittent damage of the volar sensors during cast removal.

Thirty short-arm casts were applied to the arm models by one of the authors (NCS). For one of the two secondary aims, we decided a short-arm model would provide an effective and economic measurement to assess whether the safety strip would prevent release of cast pressure on cast splitting. Ten casts in each of three groups were assessed: without a safety strip, with a safety strip on top of the four layers of padding, and with a safety strip in the middle of the padding layers. All casts were removed by one of the authors (NCS). Force measurements were recorded for all casts after initial cast application, univalving, bivalving, and after cast removal (Fig. 5C, D).
Force data were transformed to pressure by dividing by the sensor area. For each cast, the average pressure was calculated during each period of interest for approximately 30 seconds. A repeated measures ANOVA was used to determine significance $(p<0.05)$. Owing to the three pairwise t-tests among the three strip conditions, a Holm adjustment was made to the $\mathrm{p}$ values.

\section{Results}

\section{Blade-to-skin Contact Model}

Use of the safety strip placed between the padding layers decreased the number of simulated skin touches compared with casts without the safety strip present among experienced users (mean, 9.0 [range, 1-28] versus 0.1 [range, 0-1], mean ratio, 0.012 ; 95\% CI, 0.002-0.063; p < 0.001) and inexperienced users (mean, 8.5 [range, 0-31] versus 0.6 [range, 0-3], mean ratio, 0.07; 95\% CI, 0.03-0.15; p < 0.001) (Fig. 6). Use of the safety strip placed on top of the cotton padding also showed a reduction in the number of simulated skin touches compared with casts without the safety strip present (Fig. 6).

With the numbers available, while the mean number of simulated touches without the safety strip present was 


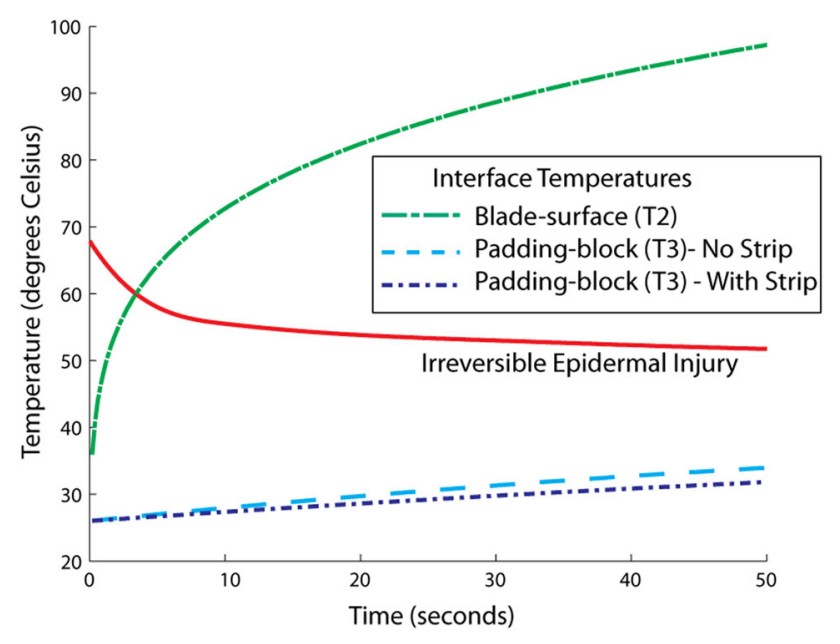

Fig. 7 A time-temperature graph shows that even at high cast saw temperatures, there is minimal heat transfer. Temperatures under the padding are less than the threshold for irreversible epidermal injury (solid red line). independent of experience (mean, 9.0 [range, 1-28] versus 8.5 [range, 0-31], mean ratio, 0.94; 95\% CI, 0.38-2.32; $\mathrm{p}=$ 0.893), fewer simulated touches were noted for experienced users compared with inexperienced users with the safety strip placed between the padding layers (mean, 0.1 [range, 0-1] versus 0.6 [range, 0-3], mean ratio, 5.89; 95\% CI, 1.44 $24.12 ; \mathrm{p}=0.020)$. Use of the safety strip placed on top of the cotton padding showed a greater difference in the reduction of simulated touches in the experienced versus inexperienced users (mean, 0.1 [range, 0-1] versus 3.0 [range, 0-10], mean ratio, 34.2; 95\% $\mathrm{CI}, 7.1-165.4 ; \mathrm{p}=0.001)$. Although the majority of touches was less than 1 second, a significant decrease was observed in the average number of touches lasting greater than 1 second when the safety strip was placed between the cotton padding layers, from 0.86 (range, $0-11$ ) to 0.03 (range, $0-1$ ) with the safety strip present (mean ratio, 0.018; 95\% CI, 0.008-0.039; $\mathrm{p}<0.001$ ). The infrequency of touches lasting greater than 1 second precluded analysis of individual conditions based on experience level.
Fig. 8A-B (A) The pressures (in $\mathrm{kPa}$ ) beneath the cast after cast placement, univalving, and bivalving for all three conditions (no strip, strip placed between the cotton padding [Within], and strip placed on top of the cotton padding [Top]) are shown. (B) There was no difference in the percent change in cast pressure after univalving or bivalving. $\mathrm{n}=$ number of casts.
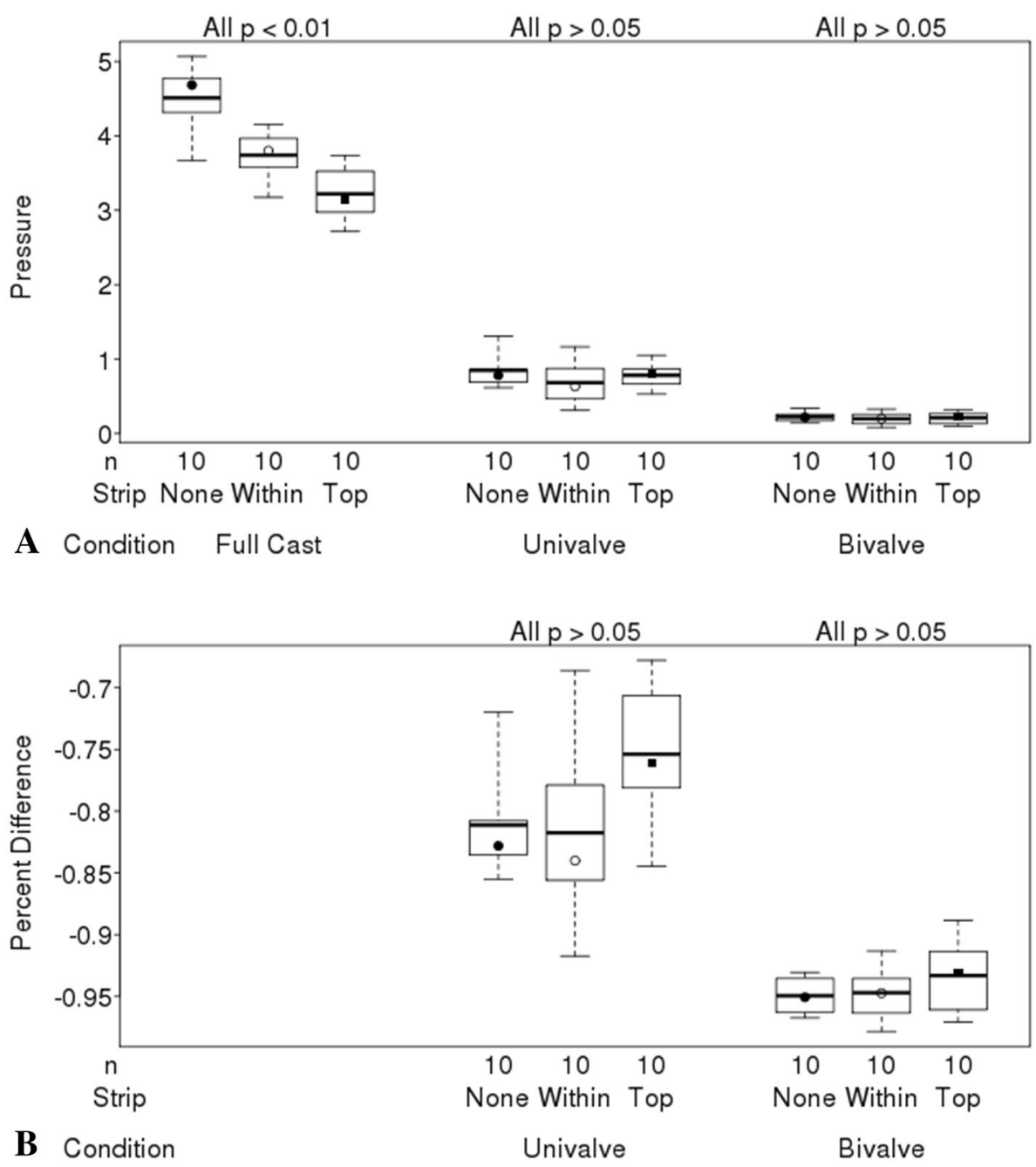


\section{Thermal Conduction}

Heat transfer was minimal through the cotton padding and safety strip (Fig. 7). Temporal data were well described by best-fit curves (T2-power law $\mathrm{R}^{2}=0.66, \mathrm{~T} 3$-second order polynomial with strip $R^{2}=0.81$, no strip $R^{2}=0.80$ ). Temperatures recorded under the padding did not exceed $40{ }^{\circ} \mathrm{C}$ when the blade temperatures were $50{ }^{\circ} \mathrm{C}$ and $65^{\circ}$ to $75{ }^{\circ} \mathrm{C}$ and did not approach $50{ }^{\circ} \mathrm{C}$ with a $100{ }^{\circ} \mathrm{C}$ blade (Fig. 7) [4, 10].

\section{Pressure Measurement}

With the numbers available, the safety strip did not affect the pressure beneath the cast after splitting the cast. Starting pressures before splitting the cast were greater in casts without the safety strip present compared with casts with the safety strip present (mean, $4.51 \mathrm{kPA}$ [SD, 0.515] versus $3.74 \mathrm{kPa}$ [SD, 0.372], mean difference, 0.77; 95\% CI, 0.38-1.16; $\mathrm{p}=0.002$, versus $3.22 \mathrm{kPa}$ [SD, 0.386], mean difference, 1.29; 95\% CI, 0.90-1.68; $\mathrm{p}<0.001$, respectively) (Fig. 8A). Placement of the safety strip between the padding layers resulted in higher starting pressures compared with casts with the safety strip placed on top of the padding (mean, $3.74 \mathrm{kPa}$ [SD, 0.372] versus $3.22 \mathrm{kPa}[\mathrm{SD},<0.386]$, mean difference, $0.52 ; 95 \% \mathrm{CI}$, $0.13-0.91 ; \mathrm{p}=0.007)$. Pressures decreased similarly regardless of strip location (Fig. 8).

\section{Discussion}

Oscillating cast saws are commonly used for splitting and removal of casts, however, they can cause iatrogenic thermal and abrasive injury to the skin. A recent study illustrated blade-to-skin contact occurs commonly with the use of the oscillating cast saw independent of experience level [9]. A higher incidence of abrasive injuries suggests that blade-to-skin contact poses a real risk of injury during cast splitting and removal. Although helpful, education and training may not be enough to prevent these events, and prevention of blade-to-skin contact may be the most effective method in reducing these iatrogenic injuries. We found that a safety strip incorporated in the cast construct resulted in fewer simulated blade-to-skin touches by the oscillating cast saw, lessened heat transfer, and did not inhibit the release of pressure that occurs when casts were univalved or bivalved. We believe the results of this experimental study justify a clinical study investigating the use of protective cast saw strips.

The primary limitation of this study is that it was conducted on a model rather than on live subjects. However, given the ethical concerns of working with humans, we created life-sized pediatric models using materials that mimic living tissue. Therefore, we believe our results can be translated to the clinical setting. The application process of the cast may raise concern for bias as poor cast application, such as inadequate padding or excessive thickness of the casting tape, can increase risk of cast saw injury. To control for this variable and potential bias, one of the authors (NCS) with experience in cast application, applied all casts in a standard fashion (four layers of padding, sixto eight-ply cast thickness). In a similar fashion, our decision to mark the projected path of the cast saw may raise concern for bias as this typically is not done in clinical practice. Our primary aim was to assess the ability of the safety strip to provide a physical barrier for the cast saw during cast removal, which it did. As such, a similar practice may be beneficial in clinical practice, although the added time to the application process may be a deterrent. A similar study could be designed to investigate if the presence of the guide marks affects the accuracy of the path of the cast saw relative to the estimation of the projected path of removal based on the visible tails of the safety strip located at either end of the cast. The location of the safety strip in the cast construct is another variable of the study. Additional study is needed to more definitively assess the potential effect of the location of the safety strip in the cast construct on blade-to-skin contact.

The decision regarding when to rotate or change cast saw blades presents a potential bias. We wanted all participants to use a blade with similar sharpness to minimize the number of confounding factors during cast removal. Although the blade could have been changed after each use, this was not thought to be economically feasible, therefore we chose to rotate and/or change after 10 casts as we believe this is a reasonable number of casts that a cast saw blade could cut without notable dullness. Future studies might investigate the role of cast saw blade maintenance on the effectiveness of the safety strip barrier. Finally, the number of inexperienced participants removing casts with the safety strip placed on top of the cotton padding versus placed between the cotton padding varied, raising concern for limitations of the study design. Initial participants showed the ability to cut through the strip when it was applied on top of the cotton padding, raising concerns that the strip had become part of the cast construct owing to the inherent stickiness of the fiberglass casting tape when wet, therefore we believe that placing the safety strip between the padding layers is important.

Another potential limitation may involve the definition of a "touch." In using a threshold based on wet materials, we might have underestimated the number of touches. Owing to the ability for water to conduct voltage, we thought it was important to use a threshold based on wet 
materials so as not to overestimate the number of touches. Despite a threshold based on wet materials and likely more-conservative definition of a "touch," a difference was detected in the presence of the safety strip. Thus, one would expect that if the number of touches were underestimated, a decrease in the number of touches would still be expected in the presence of the safety strip, however the difference may be greater in comparison. Finally, a sample of convenience was used for the various aims of the study. In the blade-to-skin contact model, a greater number of inexperienced participants was thought to be representative of various practice settings (ie, emergency department, operating room, and clinics) where cast removal is commonly performed by various healthcare personnel of different, often inexperienced, training backgrounds. The results of this model showed a difference, suggesting appropriate power. Our secondary aim, to assess whether the safety strip would inhibit release of pressure under the cast on splitting, did not show a difference on splitting, raising concern regarding whether this part of the study was adequately powered to show a difference. A difference was appreciated in the starting pressures before splitting the casts, suggesting adequate power to detect a difference between the groups.

Our study showed that a commercially available safety strip provides an effective physical barrier during splitting of a standard pediatric long-arm fiberglass cast by reducing the number of simulated touches by the oscillating cast saw.

By decreasing the number of touches, there should be fewer injurious events. Previous studies have focused on reducing cast saw blade temperatures in hopes of decreasing thermal injury associated with the oscillating cast saw $[6,11,13]$. However, the incidence of cast saw abrasions is greater than the incidence of thermal burns. One study showed that blade-to-skin contact is common during removal of casts independent of experience level, suggesting that training and education may not be enough to reduce the incidence of these events [9]. While others have suggested the use of a rubber stick inserted between the cast and the skin at the time of removal [3], their clinical use in practice can be cumbersome. To our knowledge, the concept of a safety strip incorporated in the cast construct has not been investigated before. This experimental study provides a foundation for future clinical studies. Our current study and that of Monroe et al. [9] showed that the level of training does not appear to affect the number of simulated touches alone. However, use of the safety strip led to a greater decrease in simulated touches in experienced compared with inexperienced participants, suggesting that experience and proper technique might maximize the protective effect of the safety strip. This provides another question for future research: can a combination of education and use of a safety strip further reduce cast saw injury?

Additionally, the strip lessened heat transfer to the skin of the model, keeping skin exposure temperatures below those known to cause thermal cell damage [4, 10]. This experimental setup was meant to replicate heat transfer through intact layers of cast material. However, the integrity of the strip was violated occasionally, reinforcing that no measure is completely foolproof.

The safety strip did not inhibit cast separation, suggesting that the safety strips did not prevent release of pressure when the casts were split. Pressure measurements using our model were similar to the average reported pressures beneath fiberglass casts applied to a prosthetic model [2]. These pressure measurements also were similar to in vivo pressure measurements [7,8]. Casts with a safety strip between the cotton padding layers had a lower starting mean pressure than casts without a safety strip. Casts with a safety strip on top of the cotton padding had a lower starting mean pressure compared with casts with a safety strip placed between the cotton padding layers. The presence of the safety strip on top of the cotton padding may have created a relatively uneven surface affecting application of the fiberglass, resulting in this difference. In addition, the presence of an added layer in the cast construct raises concern that this may result in uneven pressure to the underlying skin or potentially cause wrinkling of the padding, putting the patient at risk for pressure sores. Future clinical studies should investigate the potential risks involved with use of such a safety strip.

Cast saw injuries are iatrogenic complications that continue to occur despite efforts to prevent them. Interventions have focused on preventing thermal injury, however blade-to-skin contact also plays an important role in the incidence of cast saw injuries. Thus, a barrier preventing blade-to-skin contact may prove to be the most effective method to reduce injury. Although the safety strips did not eliminate blade-to-skin contact, our experimental study showed that these safety strips might provide a simple and effective tool to reduce potentially injurious cast saw injuries by decreasing touches, lessening heat transfer while not inhibiting splitting of the cast. Future studies should focus on the clinical utility and application of the protective strip. In this study, we focused on the use of such a strip with long-arm casts; however, future studies also should include various other upper and lower extremity casts. In addition, other areas of future research should include whether the safety strip has other risks associated with its use and loss of reduction in lessstable fractures or the development of pressure sores.

Acknowledgments We thank the volunteers who participated in this study. In addition, we thank Scott Hetzel MS (Department of 
Biostatistics and Medical Informatics, University of WisconsinMadison, Madison, WI, USA), for his work and preparation of the data analysis. We also thank Sarah Sund BS (Department of Orthopedics and Rehabilitation, University of Wisconsin School of Medicine and Public Health, Madison, WI, USA), for her time and work with coordination of this project.

\section{References}

1. Ansari MZ, Swarup S, Ghani R, Tovey P. Oscillating saw injuries during removal of plaster. Eur J Emerg Med. 1998;5:37-39.

2. Davids JR, Frick SL, Skewes E, Blackhurst DW. Skin surface pressure beneath an above-the-knee cast: plaster casts compared with fiberglass casts. J Bone Joint Surg Am. 1997;79:565-569.

3. Goksin C, Gunal I. Protection of the skin during cast removal. J Orthop Trauma. 1998;12:435.

4. Halanski MA, Halanski AD, Oza A, Vanderby R, Munoz A, Noonan KJ. Thermal injury with contemporary cast-application techniques and methods to circumvent morbidity. J Bone Joint Surg Am. 2007;89:2369-2377.

5. Halanski MA, Noonan KJ. Cast and splint immobilization: complications. J Am Acad Orthop Surg. 2008;16:30-40.

6. Killian JT, White S, Lenning L. Cast-saw burns: comparison of technique versus material versus saws. J Pediatr Orthop. 1999;19:683-687.
7. Maag AL, Laufer S, Kwan C, Cohen ER, Lenhart RL, Stork NC, Halanski MA, Pugh CM. Sensor-based assessment of cast placement and removal. Stud Health Technol Inform. 2014;196:259-261.

8. Mohler LR, Pedowitz RA, Byrne TP, Gershuni DH. Pressure generation beneath a new thermoplastic cast. Clin Orthop Relat Res. 1996;322:262-267.

9. Monroe KC, Sund SA, Nemeth BA, Noonan KJ, Halanski MA. Cast-saw injuries: assessing blade-to-skin contact during cast removal. Does experience or education matter? Phys Sportsmed. 2014;42:36-44.

10. Moritz AR, Henriques FC. Studies of thermal injury: II. The relative importance of time and surface temperature in the causation of cutaneous burns. Am J Pathol. 1947;23:695720.

11. Puddy AC, Sunkin JA, Aden JK, Walick KS, Hsu JR. Cast saw burns: evaluation of simple techniques for reducing the risk of thermal injury. $J$ Pediatr Orthop. 2014;34: e63-66.

12. Shore BJ, Hutchinson S, Harris M, Bae DS, Kalish LA, Maxwell W 3rd, Waters P. Epidemiology and prevention of cast saw injuries: results of a quality improvement program at a single institution. J Bone Joint Surg Am. 2014;96:e31.

13. Shuler FD, Grisafi FN. Cast-saw burns: evaluation of skin, cast, and blade temperatures generated during cast removal. $J$ Bone Joint Surg Am. 2008;90:2626-2630. 